BULL. AUSTRAL. MATH. SOC.

VOL. $33(1986), 67-70$.

\title{
A SIMPLE PROOF OF THE ERDOS-GALLAI
}

\section{THEOREM ON GRAPH SEQUENCES}

\author{
S.A. CHOUduM
}

A centrai theorem in the theory of graphic sequences is due to

P. Erdos and T. Gallai. Here, we give a simple proof of this

theorem by induction on the sum of the sequence.

THEOREM (Erdos and Gallai [2]) :

A sequence $\pi: d_{1} \geq d_{2} \geq \ldots \geq d_{p}$ of non-negative integers, whose sum (say s) is even is graphic if and only if

(EG): $\sum_{i=1}^{k} a_{i} \leq k(k-1)+\sum_{i=k+1}^{p} \min \left(d_{i}, k\right)$, for every $k, 1 \leq k \leq p$.

The known direct proofs are lengthy (see Harary [3]) while short proofs use the theory of flows in networks (see Berge [1]). Here, we give a simple direct proof. Since the necessary part is easy (see Harary [3]) we prove only sufficiency.

Proof. By induction on $s$. The theorem holds when $s=0$ or 2 . Suppose that the theorem is true for sequences whose sum is $s-2$ and let $\pi: d_{1} \geq d_{2} \geq \ldots \geq d_{p}$ be a sequence whose sum $s$ is even and which

Received 2 May, 1985. The author sincerely thanks Dr. T.V.S. Jagannathan for his valuable comments on the proof.

Copyright Clearance Centre, Inc. Serial-fee code: 0004-9727/86 $\$ A 2.00+0.00$. 
satisfies (EG). There is no loss of generality in assuming $d_{p} \geq 1$. Let $t(\geq 1)$ be the smallest integer such that $d_{t}>d_{t+1}$; if $\pi$ is regular then define $t$ to be $p-1$. Consider the sequence $\pi^{\star}: d_{1} \geq \ldots \geq d_{t-1}>d_{t}-1 \geq d_{t+1} \geq \ldots \geq d_{p-1}>a_{p}-1$. We verify that $\pi^{*}$ satisfies (EG). So, let $k$ be an integer such that $1 \leq k \leq p$. We split the proof into five cases and prove in each case that $\pi^{*}$ satisfies (EG); we use repeatedly the inequality: $\min (a, b)-1 \leq \min (a-1, b)$.

(1) $k \geq t$.

$$
\begin{aligned}
\sum_{i=1}^{k} d_{i}-1 & \leq k(k-1)+\sum_{j=k+1}^{p} \min \left(d_{j}, k\right)-1 \quad[\text { by (EG)] } \\
& \leq k(k-1)+\sum_{j=k+1}^{p-1} \min \left(d_{j}, k\right)+\min \left(d_{p}-1, k\right) .
\end{aligned}
$$

(2) $1 \leq k \leq t-1$ and $d_{k} \leq k-1$.

$$
\text { clearly, } \sum_{i=1}^{k} d_{i}=k d_{k} \leq k(k-1)+\sum_{j=k+1}^{p} \min \left(d_{j}, k\right) \text {. }
$$

(3) $1 \leq k \leq t-1$ and $d_{k}=k$.

We first observe that $d_{k+2}+\ldots+d_{p} \geq 2$. This is obvious if $k+2 \leq p-1$. If $k+2 \geq p$, then $t=p-1$ and so $\pi$ is $(p-2)^{p-1}, d_{p}$. But then, $s=(p-2)(p-1)+d_{p}$ is even, and hence $d_{p} \geq 2$. so,

$$
\begin{aligned}
\sum_{i=1}^{k} d_{i} & =k^{2}-k+d_{k+1} \leq k^{2}-k+d_{k+1}+d_{k+2}+\ldots+d_{p}-2 \\
& \leq k(k-1)+\sum_{j=k+1, \neq t}^{p-1} \min _{j}\left(d_{j}, k\right)+\min \left(d_{t}-1, k\right)+\min \left(d_{p}-1, k\right) .
\end{aligned}
$$

(4) $1 \leq k \leq t-1, d_{k} \geq k+1$, and $d_{p} \geq k+1$.

$$
\sum_{i=1}^{k} d_{i} \leq k(k-1)+\sum_{j=k+1}^{p} \min \left(d_{j}, k\right) \quad \text { [by (EG)] }
$$




$$
\begin{aligned}
=k(k-1)+\sum_{j=k+1, f t}^{p-1} \min _{j}\left(d_{j}, k\right)+ & \min \left(d_{t}-1, k\right)+\min \left(d_{p}-1, k\right) . \\
& \text { (since, } \left.\min \left(d_{j}, k\right)=\min \left(d_{j}-1, k\right)=k\right) .
\end{aligned}
$$

(5) $1 \leq k \leq t-1, d_{k} \geq k+1$ and $d_{p}<k+1$.

Let $r$ be the smallest integer such that $d_{t+r+1} \leq k$. If

$\sum_{i=1}^{k} d_{i}=k(k-1)+\sum_{i=k+1}^{p} \min \left(d_{j}, k\right)$, then we arrive at a contradiction to (EG)

as follows.

We first have,

$k d_{k}=\sum_{i=1}^{k} d_{i}=k(k-1)+(t+r-k) k+\sum_{j=t+r+1}^{p} d_{j}=k(t+r-1)+\sum_{j=t+r+1}^{p} d_{j}$.

So,

$$
\begin{aligned}
\sum_{i=1}^{k+1} d_{i} & =(k+1) d_{k}=(k+1)(t+r-1)+\frac{k+1}{k} \sum_{j=t+r+1}^{p} d_{i} . \\
& >(k+1) k+(t+r-k-1)(k+1)+\sum_{j=t+r+1}^{p} d_{j},\left(\text { since } \frac{1}{k} \sum_{j=t+r+1}^{p} d_{j}>0\right) \\
& =(k+1) k+\sum_{j=k+2}^{p} \min \left(d_{j}, k+1\right) .
\end{aligned}
$$

Hence,

$$
\begin{aligned}
\sum_{i=1}^{k} d_{i} & \leq k(k-1)+\sum_{j=k+1}^{p} \min \left(d_{j}, k\right)-1 \quad[\text { by }(\mathrm{EG})] \\
& \leq k(k-1)+\sum_{j=k+1, \neq t}^{p-1} \min \left(d_{j}, k\right)+\min \left(d_{t}-1, k\right)+\min \left(d_{p}-1, k\right) .
\end{aligned}
$$

Thus in each case $\pi^{\star}$ satisfies (EG) and hence by the induction hypothesis it is graphic. Let $G$ be a realization of $\pi^{*}$ an the vertices $v_{1}, v_{2}, \ldots, v_{p}$. If $\left(v_{t}, v_{p}\right) \notin E(G)$, then $G+\left(v_{t}, v_{p}\right)$ is a realization of $\pi$. So, let $\left(v_{t}, v_{p}\right) \in E(G)$. Since 
$\operatorname{deg}_{G}\left(v_{t}\right)=d_{t}-1 \leq p-2$, there is a $v_{m}$ such that $\left(v_{m}, v_{t}\right) \notin E(G)$. since $\operatorname{deg}_{G}\left(v_{m}\right) \geq \operatorname{deg}_{G}\left(v_{p}\right)$, there is a $v_{n}$ such that $\left(v_{m}, v_{n}\right) \in E(G)$ and $\left(v_{n}, v_{p}\right) \notin E(G)$. Deleting the edges $\left(v_{t}, v_{p}\right),\left(v_{m}, v_{n}\right)$ and adding the edges $\left(v_{t}, v_{m}\right),\left(v_{n}, v_{p}\right)$ we get a new realization $G^{*}$ of $\pi^{*}$ in which $v_{t}$ and $v_{p}$ are non-adjacent. Then $G^{*}+\left(v_{t}, v_{p}\right)$ is a realization of $\pi$.

\section{References}

[1] C. Berge, Grophs and Hypergraphs, (North Holland Pub. Co., Amsterdam, 1973).

[2] P. Erdos and T. Gallai, "Graphs with given degrees of vertices", Mat. Lapok, 11 (1960), 264-274.

[3] F. Harary, Graph Theory, (Addison Wesley, Reading, 1969).

School of Mathematical Sciences,

Madurai Kamaraj University,

Madurai - 625021 ,

India. 